\title{
Alternative proposal for Modal Representation of a Non-transposed Three-Phase Transmission Line with a Vertical Symmetry Plane
}

\author{
S. Kurokawa, A. J. Prado, J. Pissolato, L. F. Bovolato e R. S. Daltin
}

\begin{abstract}
The objective of this paper is to show an alternative representation in time domain of a non-transposed three-phase transmission line decomposed in its exact modes by using two transformation matrices. The first matrix is Clarke's matrix that is real, frequency independent, easily represented in computational transient programs (EMTP) and separates the line into quasi-modes $a, b$ and zero. After that, Quasi-modes a and zero are decomposed into their exact modes by using a modal transformation matrix whose elements can be synthesized in time domain through standard curve-fitting techniques. The main advantage of this alternative representation is to reduce the processing time because a frequency dependent modal transformation matrix of a three-phase line has nine elements to be represented in time domain while a modal transformation matrix of a two-phase line has only four elements. This paper shows modal decomposition process and eigenvectors of a nontransposed three-phase line with a vertical symmetry plane whose nominal voltage is $440 \mathrm{kV}$ and line length is $500 \mathrm{~km}$.
\end{abstract}

Keywords - electromagnetic transients analysis, frequency domain analysis, time domain analysism transmission line matrix methods.

\section{INTRODUÇÃO}

A linha de transmissão, em análises e estudos de transitórios eletromagnéticos em sistemas de potência, pode ser representada no domínio das fases ou no domínio dos modos. No domínio modal, uma linha polifásica de $n$ fases pode ser separada em seus $n$ modos de propagação e cada um desses modos comporta-se como uma linha monofásica. Sendo assim, pode-se dizer que no domínio modal uma linha de $n$ fases pode ser representada por $n$ linhas monofásicas, sendo cada uma independente das demais.

A vantagem da representação no domínio modal é que, nesse domínio, o acoplamento entre as fases da linha é eliminado, tornando mais fácil a obtenção da solução das equações diferenciais que descrevem o comportamento das correntes e tensões ao longo da mesma.

Sérgio Kurokawa, Faculdade de Engenharia de Ilha Solteira da Universidade Estadual Paulista (UNESP)., kurokawa@dee.feis.unesp.br.

Afonso J. Prado, Faculdade de Engenharia de Ilha Solteira da Universidade Estadual Paulista (UNESP), afonsojp@uol.br.

José Pissolato Filho, Faculdade de Engenharia Elétrica e de Computação na Universidade Estadual de Campinas (UNICAMP), pisso@dsce.fee.unicamp.br.

Luiz Fernando Bovolato, Faculdade de Engenharia de Ilha Solteira da Universidade Estadual Paulista (UNESP), bovolato@dee.feis.unesp.br.

Rodrigo Serra Daltin, Faculdade de Engenharia de Ilha Solteira da Universidade Estadual Paulista (UNESP), rsdaltin@aluno.feis.unesp.br.
A decomposição de uma linha de transmissão em seus modos de propagação é feita por meio do uso de uma matriz de transformação $\left[\mathrm{T}_{\mathrm{I}}\right]$ cujas colunas são autovetores associados aos autovalores do produto $[\mathrm{Y}][\mathrm{Z}]$, sendo que $[\mathrm{Z}]$ é a matriz de impedância longitudinal e [Y] é a matriz de admitância transversal da linha [1].

Os elementos da matriz $\left[\mathrm{T}_{\mathrm{I}}\right]$ geralmente são elementos pertencentes ao conjunto dos números complexos e são também variáveis em função da freqüência, sendo que a dependência em relação à freqüência torna difícil a representação dessa matriz diretamente no domínio do tempo [2]. No entanto, existem linhas de transmissão cuja configuração geométrica permite que os elementos da matriz $\left[\mathrm{T}_{\mathrm{I}}\right]$ sejam reais, independentes da freqüência e conseqüentemente fáceis de serem representados no domínio do tempo. Como exemplo de linhas cuja matriz $\left[\mathrm{T}_{\mathrm{I}}\right]$

possui somente elementos reais e constantes, pode-se mencionar as linhas bifásicas com plano de simetria vertical [3] e as linhas trifásicas idealmente transpostas [4].

No caso de linhas trifásicas que não possam ser consideradas idealmente transpostas mas que possuam um plano de simetria vertical, a matriz de Clarke separa as mesmas nas componentes $\alpha, \beta$ e zero. A componente $\beta$ é um modo exato enquanto que as componentes $\alpha$ e zero são acopladas mutuamente e são denominados quase-modos. Existem situações bem restritas em que o acoplamento entre tas componentes $\alpha$ e zero pode ser desconsiderado, fazendo com que essas componentes tornem-se também modos exatos da linha. Nessas condições, diz-se que a linha trifásica não idealmente transposta mas que possui um plano de simetria vertical pode ser decomposta em seus modos de propagação por meio do uso da matriz de Clarke, que é real, independente da freqüência e facilmente representada no domínio do tempo [4].

Para as situações em que a matriz de Clarke não possa ser considerada como sendo uma matriz de transformação modal de uma linha trifásica não idealmente transposta, este artigo propõe utilizar duas matrizes de transformação para decompor a linha em seus modos exatos. Inicialmente será utilizada a matriz de Clarke para decompor a linha em suas componentes $\alpha, \beta$ e zero. Em seguida deve-se utilizar uma matriz de transfomação modal adequada para desacoplar os quasemodos $\alpha$ e zero.

Deste modo, a linha trifásica será representada por uma linha monofásica (modo exato $\beta$ ) e por uma linha bifásica 
(quase-modos $\alpha$ e zero). A linha bifásica, que representa as componentes $\alpha$ e zero, pode então ser representada no domínio modal por meio do uso de uma matriz de transformação adequada.

O método proposto será aplicado em uma linha trifásica de $440 \mathrm{kV}$ não idealmente transposta.

\section{REPRESENTAÇÃO NO DOMÍNIO MODAL}

Sabe-se que as correntes e tensões ao longo do comprimento de uma linha de transmissão polifásica de $n$ fases podem ser descritas a partir da solução das seguintes equações diferenciais [3]:

$$
\begin{aligned}
& \frac{\mathrm{d}^{2}\left[\mathrm{~V}_{\mathrm{f}}\right]}{\mathrm{dx}^{2}}=[\mathrm{Z}][\mathrm{Y}]\left[\mathrm{V}_{\mathrm{f}}\right] \\
& \frac{\mathrm{d}^{2}\left[\mathrm{I}_{\mathrm{f}}\right]}{\mathrm{dx}^{2}}=[\mathrm{Y}][\mathrm{Z}]\left[\mathrm{I}_{\mathrm{f}}\right]
\end{aligned}
$$

Em (1) e (2) as matrizes [Z] e [Y] são, respectivamente, as matrizes de impedâncias longitudinais e de admitâncias transversais por unidade de comprimento da linha. Os vetores $\left[\mathrm{V}_{\mathrm{f}}\right]$ e $\left[\mathrm{I}_{\mathrm{f}}\right]$ contém, respectivamente, as tensões e correntes de fase da linha.

As equações diferenciais mostradas em (1) e (2) descrevem a linha de transmissão no domínio das fases. Estas equações são de difícil solução, uma vez que os produtos matriciais $[\mathrm{Z}][\mathrm{Y}]$ e $[\mathrm{Y}][\mathrm{Z}]$ resultam em matrizes cheias geralmente distintas.

No entanto pode-se utilizar uma transformação de similaridade [5] e transformar os produtos [Z][Y] e [Y][Z] em matrizes diagonais. Nessas condições, a solução das equações diferenciais da linha pode ser obtida mais facilmente.

Considerando uma matriz $\left[\mathrm{T}_{\mathrm{I}}\right]$ cujas colunas são autovetores associados aos autovalores do produto matricial [Y][Z] podese escrever [1]:

$$
[\lambda]=\left[\mathrm{T}_{\mathrm{I}}\right]^{-1}[\mathrm{Y}][\mathrm{Z}]\left[\mathrm{T}_{\mathrm{I}}\right]
$$

Em (3) $[\lambda]$ é uma matriz diagonal cujos elementos da diagonal principal são os autovalores do produto matricial $[\mathrm{Y}][\mathrm{Z}]$.

Manipulando a equação (3) e substituindo-a nas equações (1) e (2) pode-se obter:

$$
\begin{aligned}
& \frac{\mathrm{d}^{2}\left[\mathrm{E}_{\mathrm{m}}\right]}{\mathrm{dx}^{2}}=[\lambda]\left[\mathrm{E}_{\mathrm{m}}\right] \\
& \frac{\mathrm{d}^{2}\left[\mathrm{I}_{\mathrm{m}}\right]}{\mathrm{dx}}=[\lambda]\left[\mathrm{I}_{\mathrm{m}}\right] \\
& \text { sendo } \\
& {\left[\mathrm{E}_{\mathrm{m}}\right]=\left[\mathrm{T}_{\mathrm{I}}\right]^{\mathrm{T}}\left[\mathrm{V}_{\mathrm{f}}\right]} \\
& {\left[\mathrm{I}_{\mathrm{m}}\right]=\left[\mathrm{T}_{\mathrm{I}}\right]^{-1}\left[\mathrm{I}_{\mathrm{f}}\right]}
\end{aligned}
$$

As equações (4) e (5) são as equações diferenciais da linha no domínio modal. Diz-se que a linha polifásica de $n$ fases foi decomposta em seus $n$ modos de propagação. Uma vez que $[\lambda]$ é uma matriz diagonal, pode-se afirmar que (4) e (5) representam um sistema de $n$ linhas monofásicas desacopladas, cujas soluções são conhecidas [3].

Em (4)-(7) $\left[E_{m}\right]$ e $\left[I_{m}\right]$ são vetores com as correntes longitudinais e as tensões transversais, respectivamente, ao longo de cada modo da linha. A matriz $\left[\mathrm{T}_{\mathrm{I}}\right]^{\mathrm{T}}$ é a transposta de $\left[\mathrm{T}_{\mathrm{I}}\right]$ e $\left[\mathrm{T}_{\mathrm{I}}\right]^{-1}$ é a inversa de $\left[\mathrm{T}_{\mathrm{I}}\right]$.

Mostra-se que as grandezas de fase e de modo relacionamse por meio das seguintes equações [1]:

$$
\begin{aligned}
& {\left[\mathrm{Z}_{\mathrm{m}}\right]=\left[\mathrm{T}_{\mathrm{I}}\right]^{\mathrm{T}}[\mathrm{Z}]\left[\mathrm{T}_{\mathrm{I}}\right]} \\
& {\left[\mathrm{Y}_{\mathrm{m}}\right]=\left[\mathrm{T}_{\mathrm{I}}\right]^{-1}[\mathrm{Y}]\left[\mathrm{T}_{\mathrm{I}}\right]^{-\mathrm{T}}}
\end{aligned}
$$

Em (8) e (9), $\left[Z_{\mathrm{m}}\right]$ e $\left[\mathrm{Y}_{\mathrm{m}}\right]$ são matrizes diagonais que contém, respectivamente, as impedâncias e admitâncias modais por unidade de comprimento da linha

A Fig. 1 ilustra a representação de uma linha de transmisão de $\mathrm{n}$ fases no domínio modal.

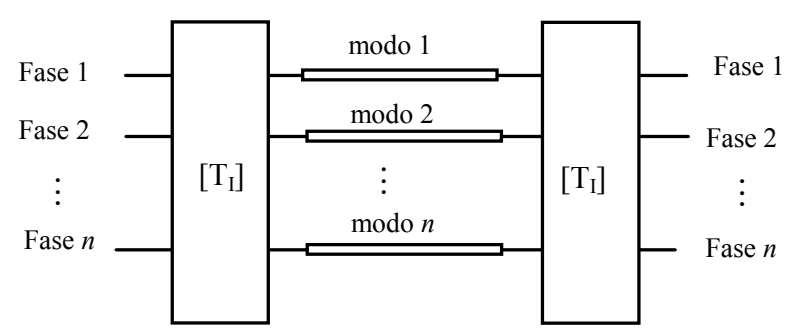

Figura 1. Representação modal de uma linha polifásica.

Na Fig. 1 as grandezas de fase são convertidas para grandezas modais através da matriz de transformação modal $\left[\mathrm{T}_{\mathrm{I}}\right]$. Em seguida, as correntes e tensões em cada um dos modos da linha são calculadas, levando-se em consideração que cada um desses modos comporta-se como uma linha monofásica sem nenhum acoplamento com os demais modos. Em outra etapa as correntes e tensões são convertidas do domínio modal para o domínio das fases.

\section{UTILIZAÇÃO DA MATRIZ DE CLARKE}

Considere uma linha de transmissão trifásica não idealmente transposta, mas que possua um plano de simetria vertical, conforme mostra a Fig. 2.

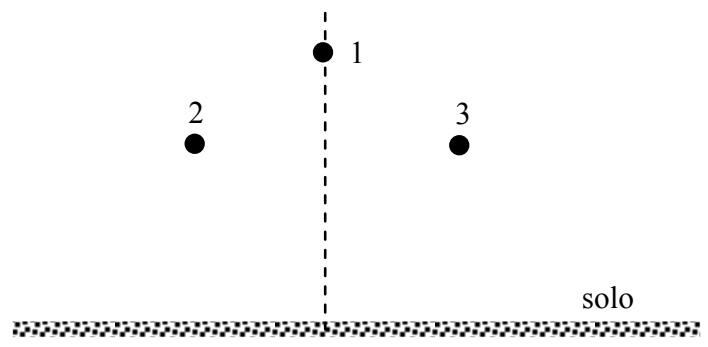

Figura 2. Silhueta de uma linha trifásica com plano de simetria vertical. 
A matriz de impedância longitudinal [Z] dessa linha apresenta a seguinte configuração [4]:

$[Z]=\left[\begin{array}{lll}A & D & D \\ D & B & F \\ D & F & B\end{array}\right]$

A matriz de admitância $[\mathrm{Y}]$ possui a mesma estrutura da matriz [Z].

Uma vez que a linha trifásica mostrada na Fig. 2 possui plano de simetria vertical, a mesma pode ser decomposta em seus quase-modos a partir do uso da matriz de Clarke como sendo uma matriz de transformação modal [2]. Substituindo-se a matriz $\left[\mathrm{T}_{\mathrm{I}}\right]$ pela matriz de Clarke, em (8) e (9), é possível obter:

$$
\begin{aligned}
& {\left[\mathrm{Z}_{\alpha \beta 0}\right]=\left[\mathrm{T}_{\text {clarke }}\right]^{\mathrm{T}}[\mathrm{Z}]\left[\mathrm{T}_{\text {clarke }}\right]} \\
& {\left[\mathrm{Y}_{\alpha \beta 0}\right]=\left[\mathrm{T}_{\text {clarke }}\right]^{-1}[\mathrm{Y}]\left[\mathrm{T}_{\text {clarke }}\right]^{-\mathrm{T}}}
\end{aligned}
$$

Em (11) e (12), a matriz de Clarke $\left[\mathrm{T}_{\text {clarke }}\right]$ pode ser escrita como:

$$
\left[\mathrm{T}_{\text {Clarke }}\right]=\left[\begin{array}{ccc}
\frac{2}{\sqrt{6}} & 0 & \frac{1}{\sqrt{3}} \\
-\frac{1}{\sqrt{6}} & \frac{1}{\sqrt{2}} & \frac{1}{\sqrt{3}} \\
-\frac{1}{\sqrt{6}} & -\frac{1}{\sqrt{2}} & \frac{1}{\sqrt{3}}
\end{array}\right]
$$

Desenvolvendo (11) e (12) obtém-se [4]:

$$
\begin{aligned}
& {\left[\mathrm{Z}_{\alpha \beta 0}\right]=\left[\begin{array}{ccc}
\mathrm{z}_{\alpha} & 0 & \mathrm{z}_{\alpha 0} \\
0 & \mathrm{z}_{\beta} & 0 \\
\mathrm{z}_{\alpha 0} & 0 & \mathrm{z}_{0}
\end{array}\right]} \\
& {\left[\mathrm{Y}_{\alpha \beta 0}\right]=\left[\begin{array}{ccc}
\mathrm{y}_{\alpha} & 0 & \mathrm{y}_{\alpha 0} \\
0 & \mathrm{y}_{\beta} & 0 \\
\mathrm{y}_{\alpha 0} & 0 & \mathrm{y}_{0}
\end{array}\right]}
\end{aligned}
$$

Observa-se que se a matriz de Clarke é utilizada como sendo uma matriz de transformação modal, a linha trifásica mostrada na Fig. 2 é separada em três componentes. As componentes $\alpha$ e zero são acopladas entre si enquanto que a componente $\beta$ é totalmente desacoplada em relação às demais.

Devido ao acoplamento mútuo, as componentes $\alpha$ e zero são denominadas quase-modos da linha e a componente $\beta$, por ser totalmente desacoplada, é um modo exato da linha. Há situações bem particulares (tais como em simulações de transitórios decorrentes de operações de manobras e chaveamentos de linhas) em que os termos mútuos $\mathrm{z}_{\alpha 0}$ e $\mathrm{y}_{\alpha 0}$ podem ser desprezados. Deste modo, as matrizes $\left[\mathrm{Z}_{\alpha \beta \beta}\right]$ e $\left[Y_{\alpha \beta 0}\right]$ podem ser escritos como sendo:

$\left[\mathrm{Z}_{\alpha \beta 0}\right] \cong\left[\begin{array}{ccc}\mathrm{z}_{\alpha} & 0 & 0 \\ 0 & \mathrm{Z}_{\beta} & 0 \\ 0 & 0 & \mathrm{z}_{0}\end{array}\right]$

$\left[\mathrm{Y}_{\alpha \beta 0}\right] \cong\left[\begin{array}{ccc}\mathrm{y}_{\alpha} & 0 & 0 \\ 0 & \mathrm{y}_{\beta} & 0 \\ 0 & 0 & \mathrm{y}_{0}\end{array}\right]$

Observa-se que se o acoplamento entre os quase-modos $\alpha \mathrm{e}$ zero for desprezado, a matriz de Clarke separa a linha trifásica mostrada na Fig. 2 em seus modos exatos.

$\mathrm{Na}$ Fig. 3 tem-se a representação esquemática da decomposição modal de uma linha trifásica considerando-se a matriz de Clarke como sendo a matriz de decomposição modal.

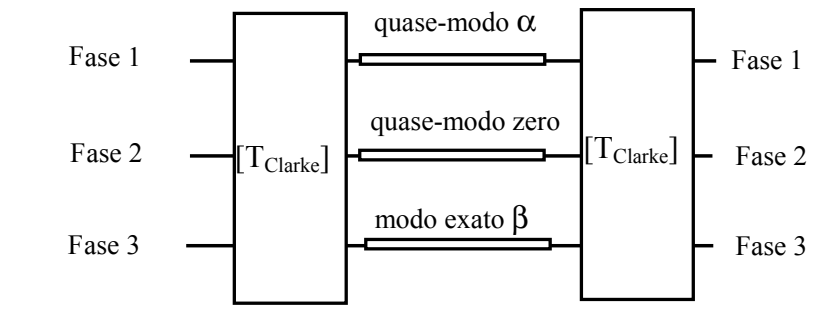

Figura 3. Decomposição modal com a matriz de Clarke.

Devido ao fato da matriz de Clarke ser uma matriz real e independente da freqüência, a mesma pode ser facilmente implementada em programas que realizam simulações de transitórios eletromagnéticos diretamente no domínio do tempo.

\section{DeComposiç̃̃o Modal Utilizando Duas Matrizes de TRANSFORMAÇ̃̃O}

\section{A. Descrição Geral}

Em situações em que a matriz de Clarke não pode ser considerada como sendo a matriz de transformação modal da linha, cuja silhueta é mostrada na Fig. 2, esse artigo propõe o uso de duas matrizes de transformação para obter os modos exatos da linha. Inicialmente utiliza-se a matriz de Clarke para obter as componentes $\alpha, \beta$ e zero. Em seguida os quase-modos $\alpha$ e zero são considerados como sendo uma linha bifásica sem plano de simetria vertical, que é então separada em seus dois modos exatos por meio do uso de uma matriz de decomposição modal $\left[\mathrm{T}_{\alpha 0}\right]$ adequada. Se os dois modos $a$ e $b$ das componentes $\alpha$ e zero tiverem as características de linhas monofásicas, os mesmos poderão ser representados por meio de modelos adequados de linhas.

A Fig. 4 mostra a representação esquemática da decomposição modal de uma linha trifásica que possui um plano de simetria vertical, por meio do uso de duas matrizes de transformação 
Na Fig. 4, $\left[\mathrm{T}_{\alpha .}\right]$ é a matriz que separa a linha bifásica (que representa os quase-modos $\alpha$ e zero) em seus modos exatos.

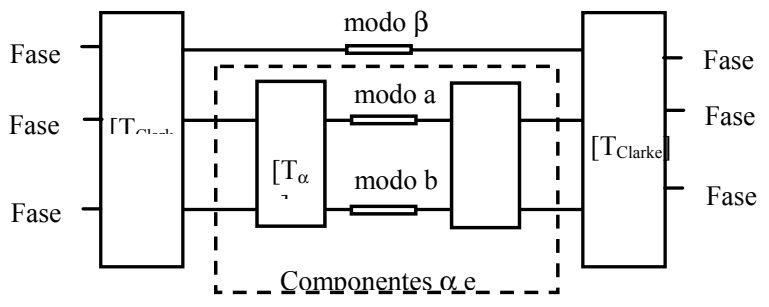

Figura 4. Decomposição modal utilizando duas matrizes de transformação.

\section{B. Decomposição Modal dos Quase-modos $\alpha$ e Zero}

Se a componente $\beta$ for excluída das matrizes $\left[Z_{\alpha \beta 0}\right]$ e $\left[Y_{\alpha \beta 0}\right]$ as mesmas tornam-se:

$$
\begin{aligned}
& {\left[\mathrm{Z}_{\alpha 0}\right]=\left[\begin{array}{cc}
\mathrm{z}_{\alpha} & \mathrm{z}_{\alpha 0} \\
\mathrm{z}_{\alpha 0} & \mathrm{z}_{0}
\end{array}\right]} \\
& {\left[\mathrm{Y}_{\alpha 0}\right]=\left[\begin{array}{cc}
\mathrm{y}_{\alpha} & \mathrm{y}_{\alpha 0} \\
\mathrm{y}_{\alpha 0} & \mathrm{y}_{0}
\end{array}\right]}
\end{aligned}
$$

As matrizes $\left[\mathrm{Z}_{\alpha 0}\right]$ e $\left[\mathrm{Y}_{\alpha 0}\right]$, mostradas anteriormente, possuem as características das matrizes de impedância longitudinal e de admitância transversal, respectivamente, de uma linha bifásica sem plano de simetria vertical do tipo mostrada na Fig. 5.

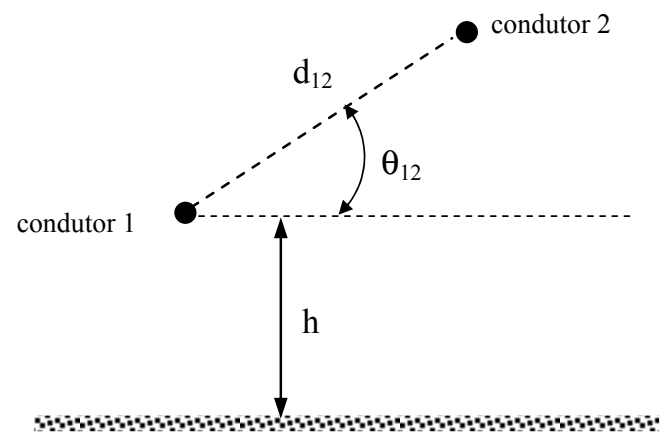

Figura 5. Linha bifásica descrita por $\left[\mathrm{Z}_{\alpha, 0}\right]$ e $\left[\mathrm{Y}_{\alpha 0}\right]$.

Na Fig. 5, os condutores 1 e 2 são os condutores de fase da linha bifásica que representa as componentes $\alpha$ e zero. $\mathrm{O}$ acoplamento entre as componentes $\alpha$ e zero é representado pelo acoplamento entre os condutores 1 e 2 .

$\mathrm{O}$ condutor 1 encontra-se a uma altura genérica $h$. Na mesma figura, $\mathrm{d}_{12}$ é a distância genérica entre os condutores 1 e 2 e $\theta_{12}$ pode assumir quaisquer valores desde que $\theta_{12} \neq 0$ e $\theta_{12} \neq \pi$. As matrizes de impedância e de admitância modais da linha, mostrada na Fig. 5, podem ser obtidas por meio das equações (8) e (9) e são escritas como sendo:

$\left[Z^{\prime}\right]=\left[\mathrm{T}_{\alpha 0}\right]^{\mathrm{T}}\left[\mathrm{Z}_{\alpha 0}\right]\left[\mathrm{T}_{\alpha 0}\right]$

$\left[\mathrm{Y}^{\prime}\right]=\left[\mathrm{T}_{\alpha 0}\right]^{-1}\left[\mathrm{Y}_{\alpha 0}\right]\left[\mathrm{T}_{\alpha 0}\right]^{-\mathrm{T}}$
Em (20) e (21), $\left[\mathrm{T}_{\alpha_{0}}\right]$ é uma matriz cujas colunas são os autovetores do produto $\left[\mathrm{Y}_{\left.\alpha_{0}\right]}\right]\left[\mathrm{Z}_{\alpha_{0}}\right]$. Essa matriz, de ordem 2, pode ser obtida por meio de métodos analíticos [5] ou numéricos [1].

Independentemente do método de cálculo utilizado para obter os autovetores, recomenda-se impor uma condição que garanta que os mesmos possuam módulo unitário. Essa condição garante que os autovetores sejam contínuos e não sofram variações abruptas em uma ampla faixa de freqüências.

Autovetores com essas características são mais facilmente representados por meio de funções racionais [1], sendo essa representação de importância fundamental quando as simulações são realizadas diretamente no domínio do tempo [6].

As matrizes $\left[\mathrm{Z}^{\prime}\right]$ e $\left[\mathrm{Y}^{\prime}\right]$ são, respectivamente, as matrizes de impedância longitudinal e de admitância transversal da linha no domínio modal e são escritas como sendo:

$\left[Z^{\prime}\right]=\left[\begin{array}{cc}z_{\mathrm{a}} & 0 \\ 0 & \mathrm{z}_{\mathrm{b}}\end{array}\right]$

$\left[Y^{\prime}\right]=\left[\begin{array}{cc}y_{a} & 0 \\ 0 & y_{b}\end{array}\right]$

Observa-se que as componentes $\alpha$ e zero, que são obtidas a partir do uso da matriz de Clarke como sendo uma matriz de transformação modal da linha trifásica são separadas em duas componentes desacopladas $a$ e $b$.

Para que tais componentes $a$ e $b$ possam ser consideradas linhas monofásicas, os elementos $\mathrm{z}_{\mathrm{a}}, \mathrm{z}_{\mathrm{b}}, \mathrm{y}_{\mathrm{a}}$ e $\mathrm{y}_{\mathrm{b}}$ devem possuir um comportamento tal que permita que os mesmos possam ser escritos na forma:

$\mathrm{z}_{\mathrm{a}}=\mathrm{R}_{\mathrm{a}}(\omega)+\mathrm{j} \omega \mathrm{L}_{\mathrm{a}}(\omega)$

$z_{b}=R_{b}(\omega)+j \omega L_{b}(\omega)$

$y_{a}=G_{a}+j \omega C_{a}$

$\mathrm{y}_{\mathrm{b}}=\mathrm{G}_{\mathrm{b}}+\mathrm{j \omega} \mathrm{C}_{\mathrm{b}}$

Em (24)-(27) os termos $R_{a}(\omega)$ e $R_{b}(\omega)$ são, respectivamente, as resistências longitudinais $\operatorname{dos} \operatorname{modos} \mathrm{a} e \mathrm{~b}$ da linha. Analogamente $L_{a}(\omega)$ e $L_{b}(\omega)$ são, respectivamente, as indutâncias longitudinais dos modos a e b da linha. Os termos $\mathrm{G}_{\mathrm{a}}$ e $\mathrm{G}_{\mathrm{b}}$ são as condutâncias transversais enquanto que $\mathrm{C}_{\mathrm{a}}$ e $\mathrm{C}_{\mathrm{b}}$ são as capacitâncias transversais dos modos da linha. Observase que, devido aos efeitos solo e pelicular, os parâmetros longitudinais da linha são variáveis em função da freqüência $\omega$ enquanto que a condutância geralmente é desprezada e a capacitância é considerada constante.

Caso os parâmetros dos modos a e b da linha bifásica, que representa os quase-modos $\alpha$ e zero, possuam as 
características descritas anteriormente, pode-se afirmar que etes modos comportam-se como sendo duas linhas monofásicas independentes caracterizadas por impedâncias longitudinais $\mathrm{z}_{\mathrm{a}}$ e $\mathrm{z}_{\mathrm{b}}$ e por admitâncias transversais $\mathrm{y}_{\mathrm{a}} \mathrm{e} \mathrm{y}_{\mathrm{b}}$.

A utilização do método de decomposição modal proposto nesse artigo pode simplificar bastante o processo de busca de uma matriz de transformação modal adequada para decompor linhas trifásicas não idealmente transpostas, mas que possuem um plano de simetria vertical, em seus modos exatos.

Essa simplificação reside no fato de que uma linha trifásica, cujas fases são acopladas, estar separada em uma linha monofásica e uma linha bifásica, sendo que essas duas linhas não possuem acoplamento entre elas. Portanto, devemos nos preocupar somente com o acoplamento entre as fases da linha bifásica. Acreditamos que essa é a maior contribuição desse artigo.

Novos estudos devem ser desenvolvidos para encontrar uma matriz de decomposição modal adequada para a linha bifásica mostrada na Fig. 5 .

\section{APLICAÇÃO DO MÉTODO}

A Fig. 6 mostra a silhueta de uma linha trifásica de $440 \mathrm{kV}$, não idealmente transposta e que possui um plano de simetria vertical. Considerou-se que a linha possui um comprimento de $100 \mathrm{~km}$.

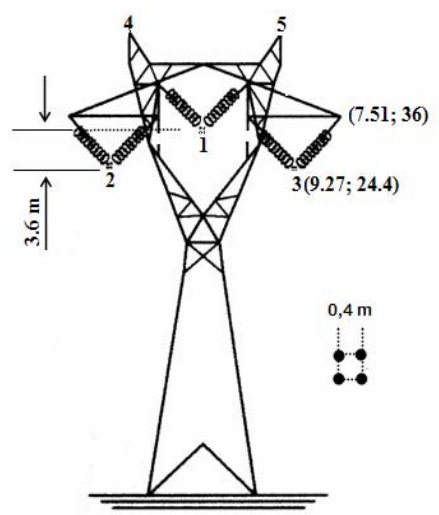

Figura 6. Silhueta de uma linha trifásica de 440 kV.

$\mathrm{Na}$ Fig. 6, as fases 1, 2 e 3 são constituídas de 4 subcondutores do tipo Grosbeak. Os condutores 4 e 5 são cabos pára-raios e são do tipo EHSW-3/8".

Os parâmetros longitudinais da linha mostrada na Fig. 6 foram calculados levando-se em consideração os efeitos solo e pelicular [7, 8]. Considerou-se que a condutância da linha é nula [9].

Para validar o processo de decomposição modal proposto, o mesmo foi aplicado na linha mostrada na Fig. 6.

\section{A. Obtenção dos modos $a$ e $b$}

Inicialmente a linha foi decomposta em suas componentes $\alpha, \beta$ e zero utilizando (11) e (12).

Em seguida, foi calculada uma matriz de transformação modal $\left[\mathrm{T}_{\alpha_{0}}\right]$ que separa os quase modos $\alpha$ e zero em seus modos exatos $a$ e $b$. Esta matriz foi obtida por meio do método de Newton Raphson [1].

As Figs. 7 e 8 mostram as partes reais e imaginárias, respectivamente, dos elementos da matriz $\left[Z^{\prime}\right]$ da linha mostrada na Fig. 6.

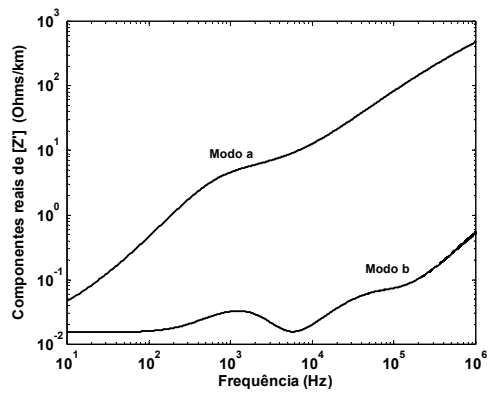

Figura 7. Componente real dos elementos da matriz $\left[Z^{\prime}\right]$.

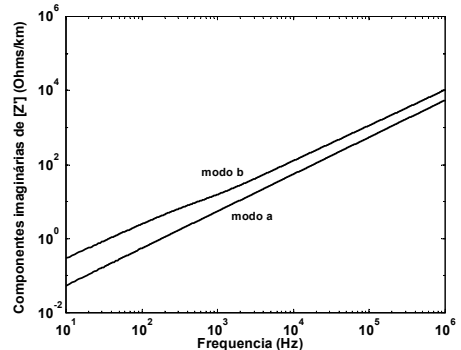

Figura 8. Componente imaginária dos elementos da matriz $\left[Z^{\prime}\right]$.

As Figs. 7 e 8 mostram que os elementos da matriz [ $\left.Z^{\prime}\right]$ possuem partes real e imaginária positivas. Conclui-se que $z_{a} e$ $\mathrm{Z}_{\mathrm{b}}$ comportam-se como impedâncias longitudinais de duas linhas monofásicas desacopladas.

A partir das componentes reais das impedâncias longitudinais dos modo a e b é possível, de acordo com (24) e (25), obter os parâmetros longitudinais dos modos a e b.

As Figs. 9 e 10 mostram, respectivamente, as resistências e indutâncias longitudinais dos modos a e b da linha bifásica que representa os quase-modos $\alpha$ e zero. Observa-se, nestas figuras, que os parâmetros longitudinais do modo b é bastante altamente dependente da freqüência. $\mathrm{O}$ mesmo não ocorre com o modo $b$, pois a resistência deste modo possui pequeno valor e sua variação com a freqüência não é tão relevante quanto à variação que ocorre no modo a. Observa-se também que a indutância do modo a é constante.

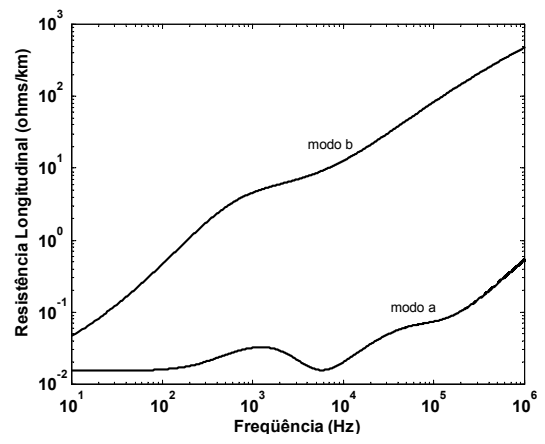

Figura 9. Resistências longitudinais dos modos a e b. 


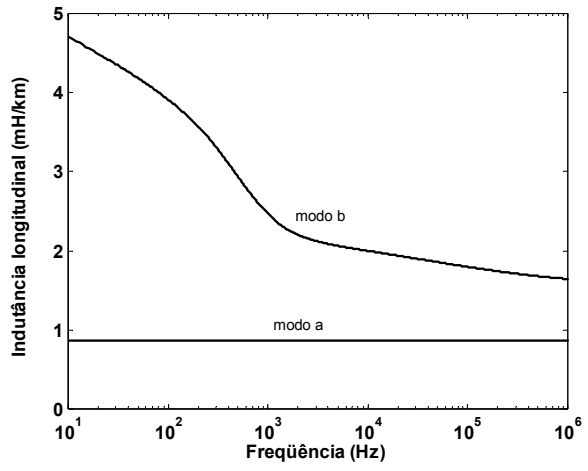

Figura 10. Indutâncias longitudinais dos modos a e b.

A Fig. 11 mostram as capacitâncias transversais dos modos a e b da linha mostrada na Fig. 6.

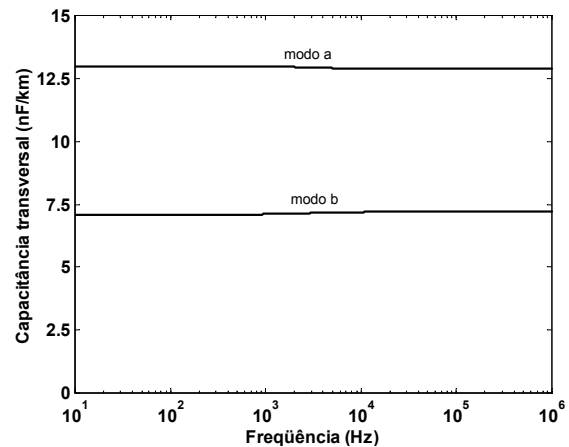

Figura 11. Resistências longitudinais dos modos a e b.

A Fig. 11 mostra que as capacitâncias dos modos a e b são constantes e que o modo $b$ possui uma capacitância maior que o modo b.

As Figs. 9-11 mostram que os parâmetros longitudinais e transversais dos modos a e b da linha mostrada na Fig. 6 representam duas linha monofásicas independentes, que podem ser modeladas por meio modelos adequados.

\section{B. Comparações}

Para comprovar que a decomposição modal proposta neste artigo está correta, a linha mostrada na Fig. 6 foi representada no domínio modal por meio de três procedimentos distintos descritos em seguida:

-Utilizando uma matriz $3 \times 3$ exata (convencional);

-Utiizando o método proposto;

-Utilizando somente a matriz de Clarke

Na primeira situação a linha foi decomposta em seus modos exatos por meio das expressões (3), (8) e (9), na segunda situação, os modos exatos da linha foram obtidos por meio do método proposto nesse artigo. Em uma terceira e última situação a linha foi decomposta em seus quase-modos por meio da matriz de Clarke, de acordo com (10)-(17).

As representações modais mencionadas anteriormente foram utilizadas para obter a resposta em freqüência da linha mostrada na Fig. 6. Nesse teste, uma fonte de tensão constante de 1 p.u. foi aplicada à fase 1 da linha, conforme mostra a Fig.
12, enquanto que os terminais emissores das fases 2 e 3 foram mantidos aterrados. Considerou-se que a linha está conectada a uma carga idêntica à impedância característica $Z_{c}$ da linha de modo a evitar que ocorram reflexões de correntes e tensões nos terminais da linha [10].

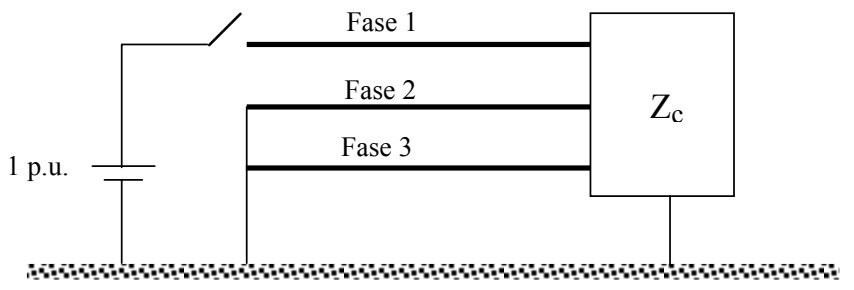

Figura 12. Energização da linha trifásica de 440 kV de 100 km.

Cada um dos modos da linha foi representado como sendo uma linha monofásica e as correntes e tensões modais foram calculadas, para cada uma das três representações modais utilizadas, por meio das soluções analíticas das equações diferenciais que caracterizam uma linha monofásica [3].

As Figs. 13 e 14 mostram o módulo das tensões nos terminais receptores das fases $1 \mathrm{e} 2$, respectivamente.

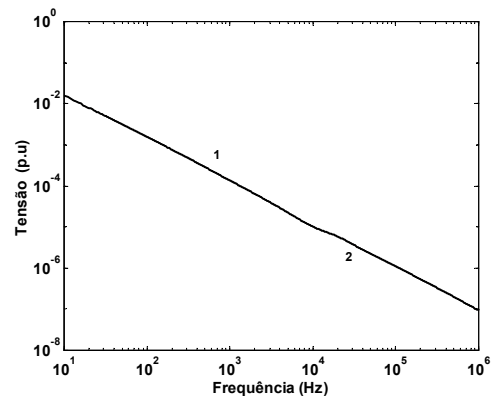

Figura 13. Módulo da tensão no terminal receptor da fase 1: Método convencional (curva 1) e método proposto (curva 2).

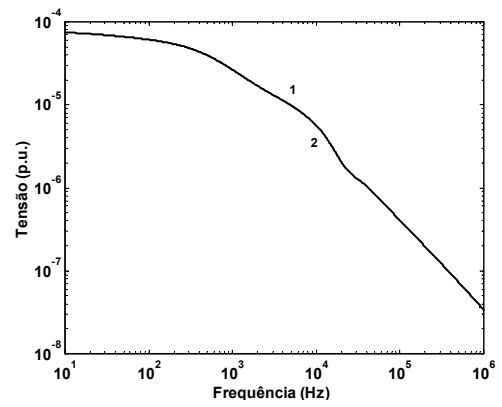

Figura 14. Módulo da tensão no terminal receptor da fase 2: Método convencional (curva 1) e método proposto (curva 2).

Nas Figs. 13 e 14, a curva 1 mostra resultados obtidos quando a linha é decomposta em seus modos exatos por meio das expressões (3), (8) e (9), sendo que nesse caso será dito que a linha foi separada em seus modos exatos por meio do método convencional. A curva 2 mostra resultados obtidos quando a linha é separada em seus modos exatos por meio do método proposto.

As Figs. 13 e 14 mostram que a resposta em freqüência obtida quando a linha é separada em seus modos exatos por meio do método convencional é idêntica à resposta obtida quando se utiliza o método proposto. Portanto conclui-se que 
o método de decomposição modal proposto nesse artigo está correto.

A Fig. 15 mostra o módulo da tensão no terminal 2 da linha, considerando os três métodos de desacoplamento modal mencionados anteriormente.

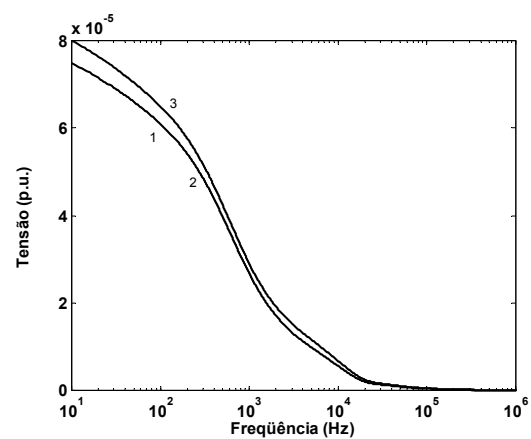

Figura 15. Módulo da tensão no terminal receptor da fase 2: Método convencional (curva 1), método proposto (curva 2) e utilizando somente a matriz de Clarke (curva 3).

A Fig. 15 mostra que o comportamento da resposta em freqüência, no terminal receptor da fase 2, quando utiliza-se os 3 métodos de decomposição modal descritos neste artigo. Observa-se que, para a situação mostrada na Fig. 12, o uso da matriz de Clarke como sendo a matriz de decomposição modal da linha produz uma amplificação da resposta em uma ampla faixa de freqüências.

Neste artigo não será feita uma abordagem a respeito da influência do erro produzido quando utiliza-se somente a matriz de Clarke, pois sabe-se que a mesma apresenta bons resultados em diversas situações já estudadas [2,4]. Neste trabalho estamos mostrando que, em situações em que a matriz de Clarke não pode ser utilizada com precisão razoável, é possível separar a linha trifásica mostrada na Fig. 6, em duas linhas de transmissão desacopladas uma em relação à outra. Neste caso, teremos uma linha monofásica e uma linha bifásica sem plano de simetria vertical.

No entanto, outros estudos devem ser realizados com o objetivo de se estudar as características desta linha bifásica. Estudos mais detalhados devem ser realizados, levando em consideração as Figs. 9-11, para que a linha bifásica possa ser modelada da maneira mais conveniente. Também não foram realizados estudos mais detalhados a respeito do comportamento da matriz que desacopla a linha bifásica nos modos exatos a e b. Estes estudos serão apresentados em trabalhos futuros.

\section{CONCLUSÕES}

Nesse trabalho foi sugerido o uso de duas matrizes de transformação para decompor uma linha trifásica, com plano de simetria vertical, em seus modos exatos de propagação.

O método consiste em, inicialmente, separar a linha em suas componentes $\alpha, \beta$ e zero utilizando a matriz de Clarke cujos elementos são números reais, independentes da freqüência e, portanto, facilmente implementados em programas que simulam transitórios eletromagnéticos diretamente no domínio do tempo. Em seguida as componentes mutuamente acopladas $\alpha$ e zero são tratadas como sendo uma linha bifásica, sem plano de simetria vertical, que pode ser decomposta em seus modos exatos por meio do uso de uma matriz de transformação modal adequada.

Os resultados obtidos mostraram que o método pode ser aplicado na linha trifásica de $440 \mathrm{kV}$, mostrada na Fig. 6.

A utilização do método de decomposição modal proposto nesse artigo pode simplificar bastante o processo de busca de uma matriz de transformação modal adequada para decompor linhas trifásicas não idealmente transpostas, mas que possuem um plano de simetria vertical, em seus modos exatos.

Essa simplificação está no fato de que uma linha trifásica, cujas fases são acopladas, é separada em uma linha monofásica e em uma linha bifásica, sendo que essas duas linhas não possuem acoplamento entre elas. Portanto, devemos nos preocupar somente com o acoplamento entre as fases da linha bifásica. Acreditamos que essa é amaior contribuição do artigo.

Novos estudos devem ser desenvolvidos para encontrar uma matriz de decomposição modal adequada para a linha bifásica mostrada na Fig. 5 .

\section{AGRADECIMENTOS}

Os autores agradecem ao Conselho Nacional de Desenvolvimento Científico e Tecnológico (CNPq) e à FAPESP (Fundação de Amparo à pesquisa) a concessão verbas para compra de equipamentos e de bolsas de estudos que possibilitaram o desenvolvimento desse trabalho.

\section{REFERÊNCIAS}

[1] L.M. Wedephol, H.V. Nguyen and G.D. Irwin, "Frequency-dependent transformation matrices for untransposed transmission lines using Newton-Raphson method", IEEE Trans. Power Delivery, vol. 11, no 3, pp. 1538-1546, Aug. 1996.

[2] M. C. Tavares, J. Pissolato and C. M. Portela, "New mode-domain multiphase transmission line for power systems studies", in Proc. Of the 1998 IEEE International Symposium on Circuits and Systems, pp. 501504.

[3] A. Budner, "Introduction of frequency-dependent line parameters into an electromagnetic transients program". IEEE Transactions on Power Apparatus and Systems, 1970, Vol. 89, nº 1, pp 88-97.

[4] M. C. Tavares, J. Pissolato and C. M. Portela, "Quasi-modes multiphase transmission line model". Electric Power Systems Research, Vol. 49, ${ }^{\circ}$ 3, pp 159-167, 1999.

[5] C. T. Chen, Linear system theory and design, CBS College Publishing, 1984.

[6] M. S. Sarto, A. Scarlatti and C. L. Holloway, "On the Use of fitting models for the time-domain analysis of problems with frequencydependent parameters", in Proc. of the 2001 IEEE Electromagnetic Compatibility International Symposium, pp. 588-593.

[7] H. W. Dommel, "Electromagnetic transients program reference manual", 1986 Department of Electrical Engineering, University of British Columbia, Vancouver, Canada.

[8] L. Marti, "Low-order approximation of transmission line parameters for frequency-dependent models", IEEE Transactions on Power Apparatus and Systems, Vol. 102, $\mathrm{n}^{\circ}$ 11, pp 3582-3589, Nov. 1983.

[9] J. A. Martinez, B. Gustavsen and D. Durbak, "Parameters determination for modeling system transients - Part I: Overhead lines", IEEE Trans. Power Delivery, vol. 20, nำ 3, pp. 2038-2044, July 2005.

[10] S. Minegishi, H. Echigo and R. Sato, "A method for measuring transients caused by interrupting current using a transmission Line terminated in its characteristics impedance", IEEE Transactions on Electromagnetic Compatibility, Vol. 36, n 3, pp 244-247, Aug. 1994. 
Sérgio Kurokawa é Engenheiro Elétrico e, desde 1994, atua como professor e pesquisador na Faculdade de Engenharia de Ilha Solteira da Universidade Estadual Paulista (UNESP). Concluiu o doutorado na Universidade Estadual de Campinas (UNICAMP) em 2003. Suas principais áreas de interesse são análise de transitórios eletromagnéticos em sistemas elétricos de potência e estudos de modelos linhas longas de transmissão de energia elétrica para uso em simulações de transitórios eletromagnéticos.

Afonso J. Prado é Engenheiro Elétrico graduado, em 1991, na Faculdade de Engenharia de Ilha Solteira da Universidade Estadual Paulista (UNESP), onde também conclui seu mestrado em 1995. Concluiu o doutorado na Universidade Estadual de Campinas (UNICAMP) em 2003. Atualmente atua como jovem pesquisador na Faculdade de Engenharia de Ilha Solteira da Universidade Estadual Paulista (UNESP). Sua principal área de interesse é a análise de transitórios eletromagnéticos em linhas de transmissão.

José Pissolato Filho é Engenheiro Eletricista e, desde 1979, atua como professor e pesquisador na Faculdade de Engenharia Elétrica e de Computação na Universidade Estadual de Campinas (UNICAMP). Concluiu seu doutorado em 1986 na Universidade Paul Sabatier, na França. Suas principais áreas de interesse são Engenharia de alta tensão, Transitórios eletromagnéticos e compatibilidade eletromagnética.

Luiz Fernando Bovolato é Engenheiro Elétrico e, desde 1977, atua como professor e pesquisador na Faculdade de Engenharia de Ilha Solteira da Universidade Estadual Paulista (UNESP). Concluiu o doutorado na Universidade de São Paulo (USP) em 1993. Suas principais áreas de atuação são transitórios eletromagnéticos em sistemas elétricos de potência, transmissão e distribuição de energia elétrica e engenharia de alta tensão.

Rodrigo Serra Daltin é Engenheiro Eletricista graduado, em 2003, na Faculdade de Engenharia de Ilha Solteira (UNESP), onde também conclui seu mestrado em 2006. 